\title{
Effects of Human Adipose-Derived Stem Cells on the Survival of Rabbit Ear Composite Grafts
}

\author{
Chae Min Kim ${ }^{1}$, Joo Hyun $\mathrm{Oh}^{2}$, Yeo Reum Jeon ${ }^{1}$, Eun Hye Kang ${ }^{2}$, Dae Hyun Lew ${ }^{2}$ \\ ${ }^{I}$ Department of Plastic and Reconstructive Surgery, National Health Insurance Service Ilsan Hospital, Goyang; ${ }^{2}$ Institute for Human Tissue \\ Restoration, Department of Plastic and Reconstructive Surgery, Severance Hospital, Yonsei University College of Medicine, Seoul, Korea
}

Background Composite grafts are frequently used for facial reconstruction. However, the unpredictability of the results and difficulties with large defects are disadvantages. Adiposederived stem cells (ADSCs) express several cytokines, and increase the survival of random flaps and fat grafts owing to their angiogenic potential.

Methods This study investigated composite graft survival after ADSC injection. Circular chondrocutaneous composite tissues, $2 \mathrm{~cm}$ in diameter, from $15 \mathrm{New}$ Zealand white rabbits were used. Thirty ears were randomly divided into 3 groups. In the experimental groups (1 and 2), ADSCs were subcutaneously injected 7 days and immediately before the operation, respectively. Similarly, phosphate-buffered saline was injected in the control group just before surgery in the same manner as in group 2. In all groups, chondrocutaneous composite tissue was elevated, rotated 90 degrees, and repaired in its original position. Skin flow was assessed using laser Doppler 1, 3, 6, 9, and 12 days after surgery. At 1 and 12 days after surgery, the viable area was assessed using digital photography; the rabbits were euthanized, and immunohistochemical staining for CD31 was performed to assess neovascularization.

Results The survival of composite grafts increased significantly with the injection of ADSCs $(P<0.05)$. ADSC injection significantly improved neovascularization based on anti-CD31 immunohistochemical analysis and vascular endothelial growth factor expression $(P<0.05)$ in both group 1 and group 2 compared to the control group. No statistically significant differences in graft survival, anti-CD31 neovascularization, or microcirculation were found between groups 1 and 2 .

Conclusions Treatment with ADSCs improved the composite graft survival, as confirmed by the survival area and histological evaluation. The differences according to the injection timing were not significant.

Keywords Graft survival / Stem cell transplantation / Adult stem cells
Correspondence: Dae Hyun Lew Department of Plastic and Reconstructive Surgery, Yonsei University College of Medicine, 50-1 Yonsei-ro, Seodaemoon-gu, Seoul 03722, Korea

Tel: +82-2-2228-2217

Fax: +82-2-393-6947

E-mail: dhlew@yuhs.ac

Received: 18 Apr $2017 \bullet$ Revised: 14 Jul $2017 \bullet$ Accepted: 29 Aug 2017

pISSN: 2234-6163 • elSSN: 2234-6171 • https://doi.org/10.5999/aps.2017.44.5.370 • Arch Plast Surg 2017;44:370-377

No potential conflict of interest relevant to this article was reported.

\section{INTRODUCTION}

Restoration of physical defects or deformities resulting from various congenital or acquired causes to a normal morphologi- cal and functional state is the primary aim of plastic and reconstructive surgery. It is imperative for a surgeon to consider not only functional aspects, but also aesthetic aspects, within a wide spectrum of reconstructive procedures, such as primary repairs, 
skin grafts, composite grafts, local flaps, and free vascularized flaps. The surgical method should be determined based on various factors such as the size of the defect, involvement of scar contracture, color, texture, and donor site morbidity. Composite grafts are a particularly useful tool and have a number of benefits [1]. Composite grafts result in less discrepancy in color and texture at the recipient site than skin grafts, and are associated with less scar contracture, as more structural support is provided by transferring 2 or more different types of tissues. For example, chondrocutaneous composite grafts containing cartilage tissue enable reconstruction and preservation of the 3-dimensional structure [2]. However, a drawback of composite grafts lies in the limited predictability of graft acceptance if the defect is larger than $1.5 \mathrm{~cm}$ in diameter [3].

To overcome this limitation, several complimentary methods have been proposed to improve graft survival. To date, multiple studies have advocated the validity of such techniques, including ice-cold compression, steroid injection, hyperbaric oxygen therapy, fibroblast growth factor (FGF) injection, and plateletrich plasma injection $[4,5]$. Adipose-derived stem cells (AD$\mathrm{SCs}$ ) are known to induce various growth factors, such as vascular endothelial growth factor (VEGF) and hepatocyte growth factor through paracrine activity. They also possess angiogenic potential, which enhances the survival of random flaps and grafted fat tissue through neovascularization [6-8]. Moreover, based on the findings that preconditioned ADSCs improved flap survival, and that the amount of VEGF peaked 1 week after ADSC injection $[9,10]$, we decided to apply ADSCs to composite grafts. In this study, we demonstrated the overall effect of ADSCs on the survival of composite grafts, and investigated which timing of the ADSC injection yielded the best results.

\section{METHODS}

\section{Animal model, groups, and preparation}

Circular chondrocutaneous tissue with a diameter of $2 \mathrm{~cm}$ was raised bilaterally on the ears of $15 \mathrm{New}$ Zealand white rabbits [5]. The rabbits were treated in the animal laboratory of our hospital, and the Animal Care and Experiment Committee approved the experimental protocol. Thirty chondrocutaneous samples were randomly divided into 3 groups with 10 ears in each group. In group 1, a 1.0-mL mixture of $2 \times 10^{6} \mathrm{ADSC}$ and phosphate-buffered saline (PBS) was evenly injected subcutaneously at 4 spots, $0.5 \mathrm{~cm}$ from the expected circular composite tissue graft site, using a 1.0-mL syringe 7 days before composite tissue elevation. The rabbits in group 2 underwent the same protocol except that the ADSC injection was performed immediately before the composite tissue was elevated. The control group received only $1.0 \mathrm{~mL}$ of PBS just before surgery in the same manner as group 2.

\section{Isolation and expansion of ADSCs in vitro}

ADSCs were isolated from the lipoaspirates of liposuction patients from the fatty lower abdominal area, after receiving informed consent, using Coleman's method. A solution of $0.1 \%$ collagenase (Wako, Osaka, Japan) in PBS $(20 \mathrm{~mL})$ was applied to $10 \mathrm{~g}$ of human aspirate, followed by treatment in a $37^{\circ} \mathrm{C}$ shaking incubator for 60 minutes. To prevent collagenase activity, we added $20 \mathrm{~mL}$ of medium (DMEM/F12, Invitrogen, Grand Island, NY, USA) at $4^{\circ} \mathrm{C}, 10 \%$ fetal bovine serum (FBS, Hyclone, Logan, Utah), and 1\% penicillin-streptomycin solution (Sigma Aldrich, Steinheim, Germany). The treated lipoaspirate was centrifuged at $1,200 \times \mathrm{g}$ for 10 minutes at room temperature $\left(25^{\circ} \mathrm{C}\right)$, and the stromal vascular fraction (SVF) was obtained from the bottom layer. The SVF was cultured in an inductive medium in $75 \mathrm{~T}$ flasks (Corning Inc., Corning, NY, USA) for 2 days at $37.3^{\circ} \mathrm{C}$ in a $5 \% \mathrm{CO}_{2}$ incubator. Two days later, unattached cells were washed away using PBS, leaving only attached cultured cells. The growth medium was exchanged every 3 days, and when cells reached a confluent state, they were detached using $0.25 \%$ trypsin (Gibco, NY, Gran Island, USA) solution and subcultured. In this study, we harvested ADSCs from passage 4.

\section{Surgical procedure}

Fifteen New Zealand white rabbits with an average weight of 3.0 $\mathrm{kg}$ were used, and bilateral ear chondrocutaneous composite tissues were elevated and rotated [5]. All rabbits fasted starting the night before the procedure. An intramuscular injection of $\mathrm{Zo}$ letil 50 (Virbac, Carros, France) and 2\% Rompun (Bayer, Leverkusen, Germany) (1:2 ratio, $1 \mathrm{~mL} / \mathrm{kg}$ ) was performed for anesthesia. After being shaved and draped with a povidone-iodine solution, a circular composite tissue with a 2 -cm diameter was designed $4 \mathrm{~cm}$ distal to the external meatus. A full-thickness chondrocutaneous composite graft was elevated and rotated $90^{\circ}$ counterclockwise. The graft was secured onto the site with a 6-0 nylon suture (Fig. 1).

\section{Assessment of the survival area of the composite graft}

On postoperative days $0,1,3,6,9$, and 12 , digital images of the grafts were taken under the same conditions and at equal distances (EOS 600D, Canon, Tokyo, Japan). Using the Image J software (National Institutes of Health, Bethesda, MD, USA), 2 independent reviewers quantified the ratio of viable surface area to the total graft surface area based on pixel number. Each group was compared based on the average values obtained by the reviewers. 


\section{Assessment of microcirculation with laser Doppler flowmetry}

To evaluate the changes in circulation over time, laser Doppler flowmetry (Peri-Flux System 5000, Perimed Inc., Stockholm, Sweden) was used on the rotated composite grafts preoperative-

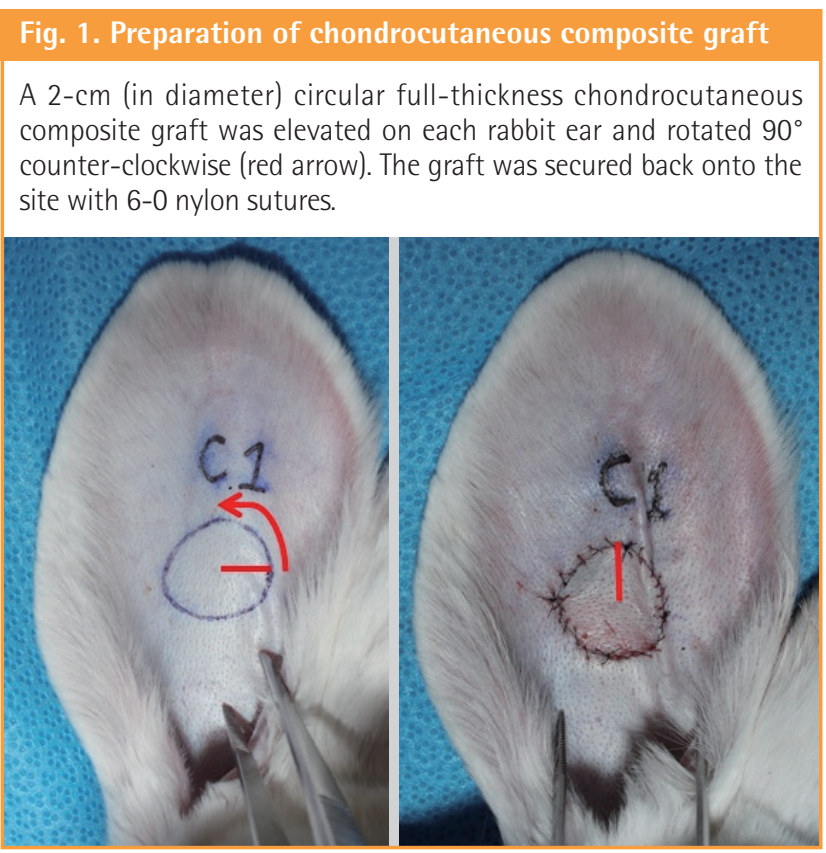

ly and on days 1, 3, 6, 9, and 12 after surgery [11]. Percutaneous blood flow was detected for at least 1 minute to ensure stable signals at 4 different spots on the graft surface area. The retrieved perfusion data was quantified and further analyzed with the Perisoft program (Perimed Inc.).

\section{Assessment by immunohistochemical analysis}

On postoperative day 12 , the rabbits were sacrificed and the viable area of the composite graft was excised to produce tissue specimens that were later embedded in paraffin. The histological slides were subjected to immunohistochemical staining using an anti-VEGF monoclonal antibody (ab1316, Abcam, Cambridge, MA, USA) and an anti-CD31 (anti-mouse platelet endothelial cell adhesion molecule-1; PECAM/CD31) polyclonal antibody (ab9498, Abcam). For anti-VEGF, semi-quantitative analysis was performed with the Metamorph image analysis software (Molecular devices, Sunnyvale, CA, USA). For endothelial cells stained with the anti-CD31 antibody, 2 independent observers quantified the degree of neovascularization using high-power microscopy $(\times 400)$.

\section{Statistical analysis}

Graphpad Prism 5 software (Graphpad software, San Diego, CA, USA) was used for statistical analysis. Analysis was per-

Fig. 2. Viability changes in composite grafts

Over time, the area of central necrosis was demarcated, and composite grafts injected with ADSCs in groups 1 and 2 retained significant viability when compared to the control group $(P<0.05)$. ADSC, adipose-derived stem cell; POD, postoperative day.

POD \#0

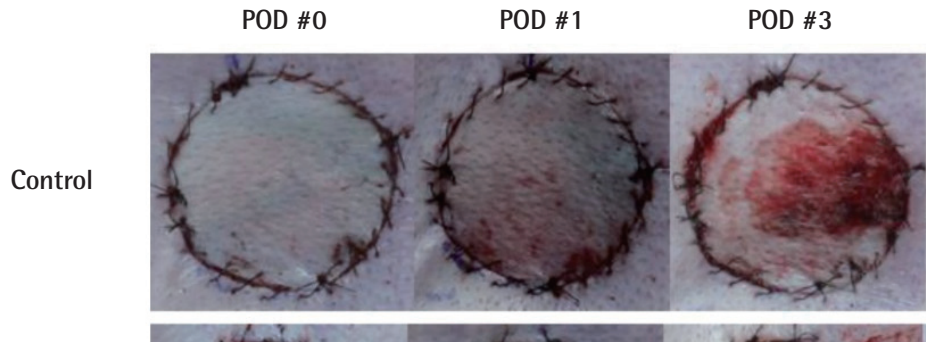

Control

Group 1

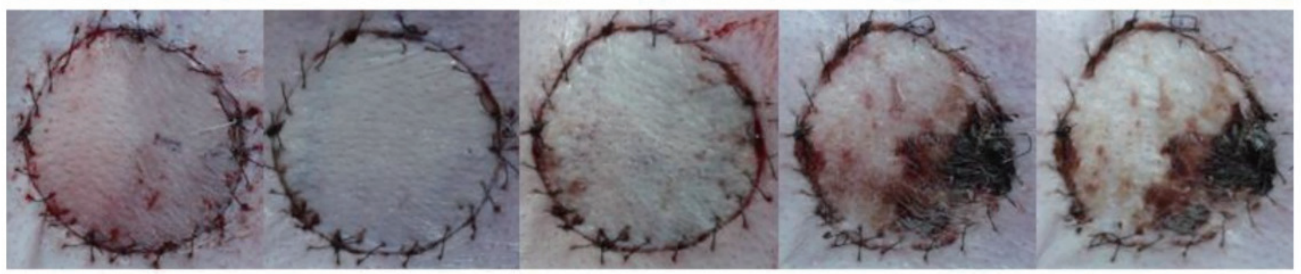

Group 2

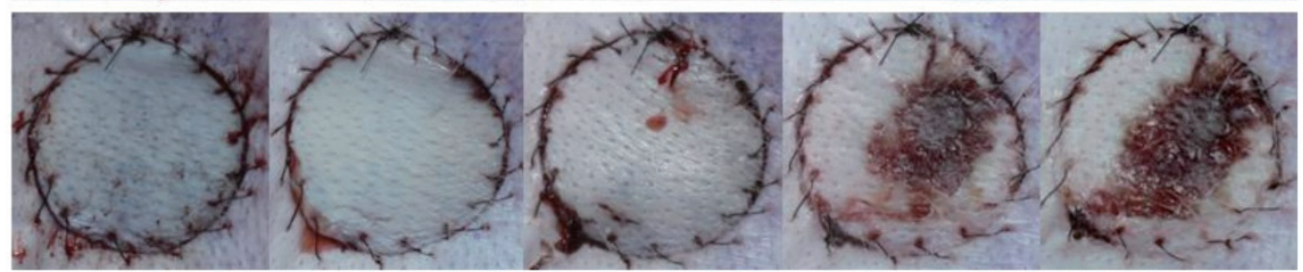


formed using analysis of variance and the Bonferroni test; differences were considered statistically significant at $\mathrm{P}<0.05$.

\section{RESULTS}

\section{Effect on composite graft survival area}

Necrotized tissue was gradually observed over time where perfusion was limited, and its location was mostly central rather than marginal (Fig. 2). The survival rates, expressed as the ratio of viable surface area to that of the entire composite graft, were $68.1 \%( \pm 9.6 \%)$ in group $1,65.4 \%( \pm 6.2 \%)$ in group 2 , and

\section{Fig. 3. Comparison of the survival area}

The survival area in ADSC-treated composite grafts was significantly larger than in the control group $\left({ }^{*} P<0.05\right)$. However, regardless of whether ADSCs were injected 7 days earlier (as in group 1) or immediately before the procedure (as in group 2), this difference was not statistically significant. ADSC, adipose-derived stem cell.

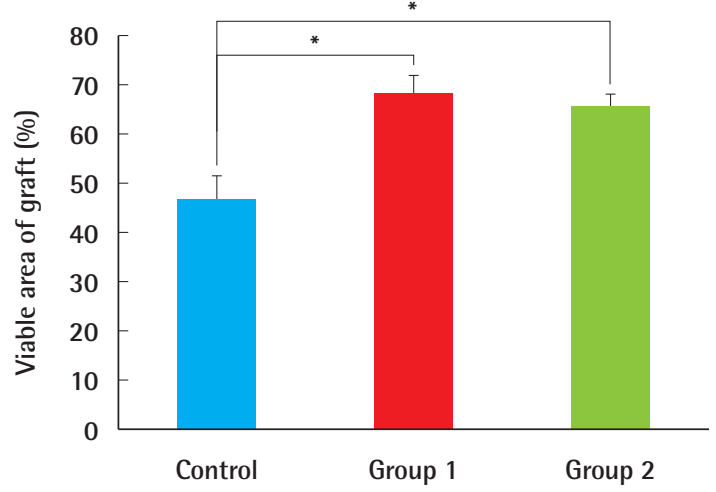

$46.83 \%( \pm 13.4 \%)$ in the control group, demonstrating the significant benefit of ADSCs in groups 1 and 2, compared to the control group $(\mathrm{P}<0.05)$. The difference between groups 1 and 2 , in which the timing of the ADSC injection differed, was not statistically significant (Fig. 3).

\section{Effect on the microcirculation of the composite graft}

Microcirculation measurements by laser Doppler flowmetry demonstrated that groups 1 and 2, which were treated with ADSC injection, had greater mean circulation values than the control, and the difference was significant 3 days after surgery $(\mathrm{P}<0.05)$. However, besides this difference, no significant difference was found. By postoperative day 12 , these values diverged, with 28.0 perfusion units $(\mathrm{PU})( \pm 9.8 \mathrm{PU})$ in group 1 and 26.4 PU $( \pm 9.81 \mathrm{PU})$ in group 2 , whereas the control group showed only a gradual increase to $17.7 \mathrm{PU}( \pm 4.8 \mathrm{PU})$ (Fig. 4).

\section{CD31-positive neovascularization in the composite graft}

The average number of newly formed blood vessels, observed using $\times 400$ magnification, was $2.2( \pm 1.3)$ neovessels in the control group, $6.9( \pm 1.9)$ in group 1 , and $6.3( \pm 2.1)$ in group 2 (Fig. 5). A statistically significant increase was found in neovascularization in the ADSC-treated grafts compared with the control group. However, no significant difference was found between group 1 and group 2, implying that the timing of the ADSC injection was not important for this parameter (Fig. 6).

VEGF expression was higher in the ADSC-treated groups 1 and 2 than in the control group (Figs. 7, 8). The composite

\section{Fig. 4. Comparison of microcirculation by laser Doppler flowmetry}

Comparison of the microcirculation level in the ADSC-treated groups with that in the phosphate-buffered saline-treated control group using laser Doppler flowmetry; the circulation appeared to be greater in the ADSC-treated grafts. In particular, statistical significance was found on postoperative (POD) day $3\left({ }^{*} \mathrm{P}<0.05\right)$. ADSC, adipose-derived stem cell.

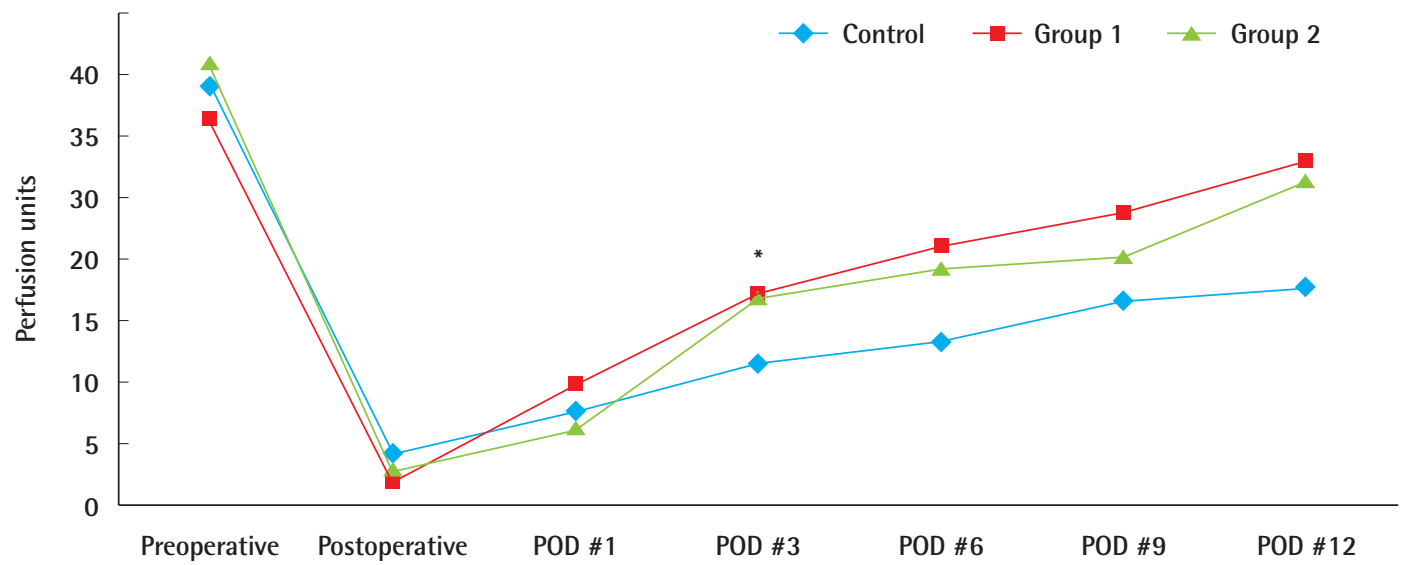




\section{Fig. 5. Immunohistochemistry with anti-CD31 antibody}

Twelve days after the procedure, the tissue specimen was subjected to immunohistochemical testing using an anti-CD31 antibody ( $\times 400)$. This demonstrated increased CD31 expression in groups 1 and 2 compared to that in the control group. (A) Control, (B) group 1, (C) group 2.
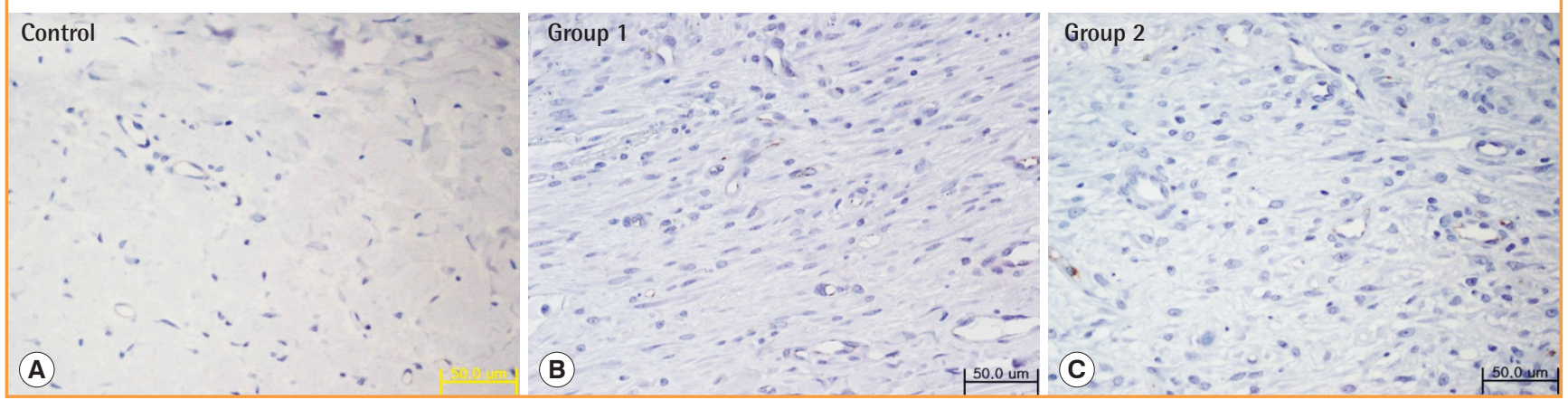

\section{Fig. 6. Neovascularization levels}

The number of newly formed vessels was greater in composite grafts after ADSC injection compared to the control group $\left.{ }^{* *} \mathrm{P}<0.01\right)$. There were no significant differences with regard to the timing of the ADSC injections. ADSC, adipose-derived stem cell; HPF, high-power field.

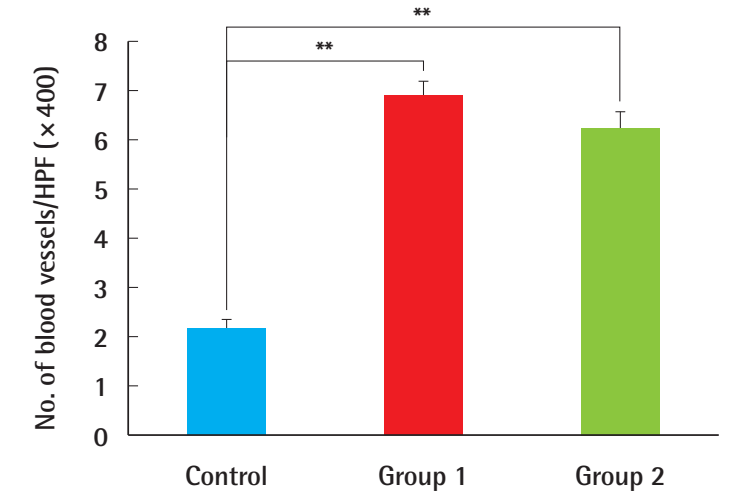

grafts in group 2, with ADSC injection performed immediately before graft elevation, showed the highest VEGF expression, followed by group 1 , in which the ADSCs were injected 1 week before surgery. The control group treated with PBS only had the lowest expression. The differences among all groups were statistically significant $(\mathrm{P}<0.01)$.

\section{DISCUSSION}

The wound healing process takes place via complex cascades of various cytokines, growth factors, and chemokines. Since stem cells are the primary source of these factors, many attempts have been made to utilize their highly interactive paracrine effect [12]. Previous animal studies have shown that stem cells exerted regenerative effects when injected into damaged tissue $[8,13]$. Numerous human studies have also reported increased viability in intractable chronic wounds $[14,15]$.

Stem cells may play a direct role in structural regeneration through differentiation into epidermal keratinocytes, endothelial cells, or pericytes. In addition, it is known that these cells exhibit a paracrine effect, resulting in the secretion of various growth factors such as FGF and VEGF, which in turn induce localized cellular reactions such as the inflammatory response, angiogenesis, and fibroproliferation $[15,16]$. Stem cells can be extracted from the bone marrow, umbilical cord blood, and adipocytes, and these cells differ in their expression profiles and characteristics depending on their source $[17,18]$. Due to the limited availability of umbilical cord blood, clinical applications using adults mainly rely on bone marrow-derived and adipose-derived stem cells, and their differentiation potentials are comparable to each other [18]. Adipose-derived stem cells are most widely used in research, as they can be obtained with minimal invasiveness compared to bone marrow-derived stem cells, and are abundant [19]. Moreover, their minimal immunogenicity and lack of allograft or xenograft rejection have made ADSCs readily applicable for human clinical studies and animal studies [20,21].

For these reasons, we decided to utilize ADSCs to enhance the survival of composite grafts, as these were once regarded as a useful option for facial reconstruction; however, their applications have also been limited due to their confined range of graft size. Currently, no method can dramatically increase the viability of composite grafts. In addition, based on a recent study in which preoperative ADSC injection enhanced the survival of random flaps [9], we applied this methodology to composite grafts. We expected that this would allow the recruitment of factors contributing to graft take, potentially affecting the neovascularization and overall survival of the graft. In this study, the groups treated with ADSCs showed a higher survival rate and more neovascularization than the control group; however, there was no difference depending on whether the ADSC injection 


\section{Fig. 7. Immunohistochemistry with anti-VEGF antibody}

Based on immunochemical staining $(\times 400)$ with an anti-VEGF monoclonal antibody, group 1 and group 2, both of which received an ADSC injections, displayed more noticeable brown-colored staining. In particular, anti-VEGF staining was most pronounced in group 2, in which the ADSCs injection was done immediately before composite graft elevation. (A) Control, (B) group 1, (C) group 2. VEGF, vascular endothelial growth factor; ADSC, adipose-derived stem cell.
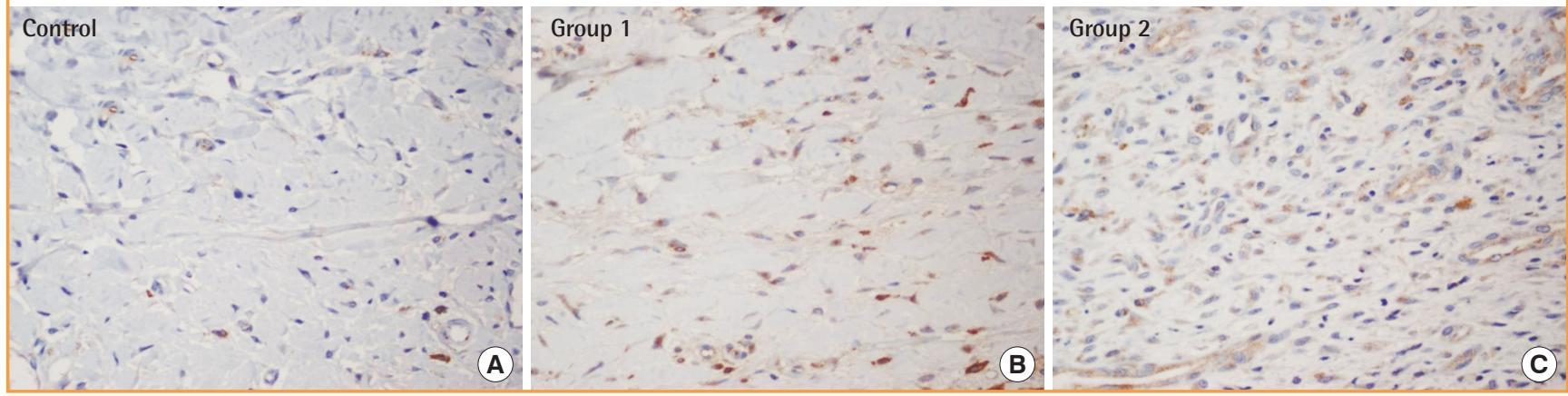

\section{Fig. 8. Relative expression of VEGF}

Based on a semi-quantitative analysis with the Metamorph image analysis software (Molecular devices, Sunnyvale, CA, USA), VEGF expression was greater in groups 1 and 2, and the difference was statistically significant between group 1 and group 2. This suggests that VEGF was expressed more abundantly when ADSCs were injected immediately before the composite graft procedure, rather than 7 days before surgery ( $\left.{ }^{* *} P<0.01\right)$. VEGF, vascular endothelial growth factor; ADSC, adipose-derived stem cell.

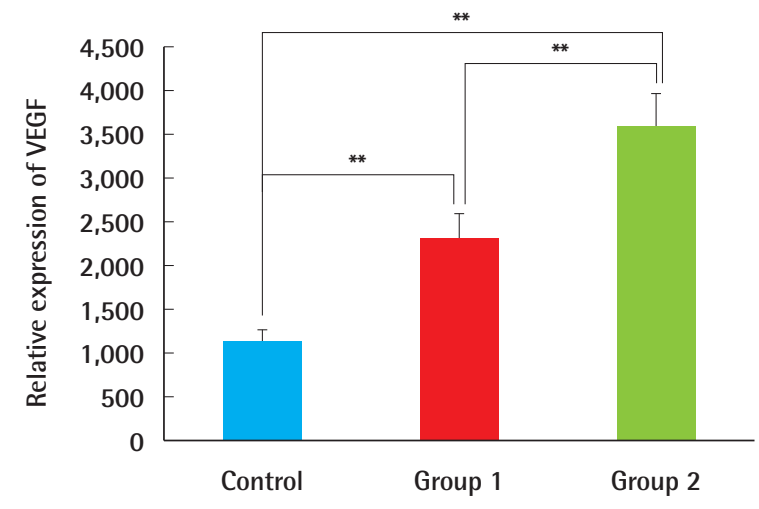

was performed shortly before surgery or several days prior. In contrast, according to Yucel et al. [22], survival rates were significantly different between the group injected with ADSCs 4 days prior to composite graft surgery and the group injected immediately before surgery. It has been speculated that the raw surface stimulates the paracrine effect of ADSCs.

One of the possible reasons for why the results seemed unrelated to the timing of the ADSC injection is that the growth factors might not have been sufficiently recruited via proper stimuli. For example, studies have shown that a hypoxic state can act as a potent stimulus for stem cells, thereby preconditioning $\mathrm{AD}$ SCs, leading to a surge in the release of various growth factors such as VEGF or basic FGF $[9,23]$. However, other studies have successfully demonstrated that ADSCs had a significant benefit on the survival of random flaps without the need for hypoxic preconditioning [9]. In addition, it was not possible to induce a hypoxic stimulus by surgical delay in composite grafts. Although graft survival might be affected by surgical skill, this effect was negligible in this study, as all surgical procedures were performed by a single plastic surgeon. The rate of complications due to seroma or hematoma did not differ significantly among the groups in this study.

Microcirculation measurements by laser Doppler flowmetry consistently showed in an increase in this parameter in rabbits treated with ADSCs compared to the control group, but this was not statistically significant except on postoperative day 3 . The reason for this may have been that the sample size was not large enough, with only 10 ears in each group, and that the microcirculation level was highly variable among individual composite grafts. Additionally, as the microcirculation appeared comparable between the $2 \mathrm{ADSC}$-treated groups, it can be assumed that ADSCs played a similar role in composite graft take, regardless of the timing of ADSC application.

Based on immunohistochemical staining with an anti-CD31 antibody, the difference in neovascularization was not statistically significant, despite the previously noted differences between the ADSC-treated groups and the control group. In this respect, ADSCs showed a positive influence on neovascularization, but the effect of early injection was not significant. The early-treated ADSCs might not have recruited growth factors in advance, but did so upon an external stimulus, which was the surgical procedure in this study.

The paracrine effect of ADSCs was previously reported to induce neovascularization by attracting growth factors such as VEGF [6], and the ADSC-treated grafts in this study showed a significant increase in VEGF secretion compared the control 
group. VEGF expression was more prominent when ADSCs were injected on the day of the operation rather than 1 week prior. This was mainly attributed to the fact that 19 days elapsed before the VEGF level was finally measured, as the graft tissue specimen was taken 12 days after surgery. It was speculated that this was sufficient time for the ADSCs to gradually lose their capacity to recruit VEGF. This also agrees with the findings of a previous study, in which in vitro cultured ADSCs showed an initial increase in VEGF secretion during the first week, which subsequently subsided [10]. Additionally, the ADSCs that were not stimulated seemed to have become inactive to a certain extent during 7 days and did not have any specific function. The exact mechanism is unknown. However, it can be inferred that some of the stem cells have undergone apoptosis. We speculate that only those surviving when stimulation occurred after surgery were involved in the expression of VEGF.

There are several limitations to consider for this study. First, the general effect of ADSCs throughout the whole body was not taken into account. ADSCs are reportedly able not only to exert local paracrine effects, but also to have general effects via intravenous injection. To rule out the unintended general impact of ADSCs and to obtain objective results, independent individual subjects should be used in a subsequent study. Second, as histologic analysis was undertaken at the end of the study, the time course of VEGF expression changes could not be closely observed. In fact, the authors were not able to confirm at which point VEGF secretion peaked or how these changes proceeded. Using more qualitative measures such as western blot or enzyme-linked immunosorbent assays would probably yield better results. In addition, since the paracrine effect of ADSCs occurs at the recipient site, it is necessary to compare the amount of VEGF secretion and histological changes in both the recipient site and surrounding tissues. Therefore, only comparing the differences at the graft sites is a limitation of this study. Third, human ADSCs were injected in our study instead of rabbit ADSCs. ADSCs have been reported to be effective in xenotransplantation because of their low immunogenicity, but for accurate experimentation, injection with rabbit ADSCs should have been performed. Moreover, in order to exclude the effect of acute inflammation, a control group in which different parts of human cells were injected should have been included. Finally, the timing of injection could have been varied to determine the optimal time to apply ADSCs. A difference of 1 week in the ADSC injection timing did not result in any statistically significant changes, although it did seem to enhance the survival of composite grafts. Thus, injection timing should be further evaluated to determine the optimal period for ADSC injection, and to assess optimal preconditioning timing.

\section{REFERENCES}

1. Cheon YW, Park BY. Long-term evaluation of elongating columella using conchal composite graft in bilateral secondary cleft lip and nose deformity. Plast Reconstr Surg 2010; 126:543-53.

2. Son D, Kwak M, Yun S, et al. Large auricular chondrocutaneous composite graft for nasal alar and columellar reconstruction. Arch Plast Surg 2012;39:323-8.

3. Jeon YR, Kang EH, Yang CE, et al. The effect of platelet-rich plasma on composite graft survival. Plast Reconstr Surg 2014;134:239-46.

4. Harbison JM, Kriet JD, Humphrey CD. Improving outcomes for composite grafts in nasal reconstruction. Curr Opin Otolaryngol Head Neck Surg 2012;20:267-73.

5. Li EN, Menon NG, Rodriguez ED, et al. The effect of hyperbaric oxygen therapy on composite graft survival. Ann Plast Surg 2004;53:141-5.

6. Rubina K, Kalinina N, Efimenko A, et al. Adipose stromal cells stimulate angiogenesis via promoting progenitor cell differentiation, secretion of angiogenic factors, and enhancing vessel maturation. Tissue Eng Part A 2009; 15:2039-50.

7. Sterodimas A, de Faria J, Nicaretta B, et al. Tissue engineering with adipose-derived stem cells (ADSCs): current and future applications. J Plast Reconstr Aesthet Surg 2010; 63:1886-92.

8. Lu F, Mizuno H, Uysal CA, et al. Improved viability of random pattern skin flaps through the use of adipose-derived stem cells. Plast Reconstr Surg 2008;121:50-8.

9. Yue Y, Zhang P, Liu D, et al. Hypoxia preconditioning enhances the viability of ADSCs to increase the survival rate of ischemic skin flaps in rats. Aesthetic Plast Surg 2013;37: 159-70.

10. Lu F, Li J, Gao J, et al. Improvement of the survival of human autologous fat transplantation by using VEGF-transfected adipose-derived stem cells. Plast Reconstr Surg 2009;124: 1437-46.

11. Choi CM, Bennett RG. Laser Dopplers to determine cutaneous blood flow. Dermatol Surg 2003;29:272-80.

12. Ko SH, Nauta A, Wong V, et al. The role of stem cells in cutaneous wound healing: what do we really know? Plast Reconstr Surg 2011;127 Suppl 1:10S-20S.

13. Nauta AC, Gurtner GC, Longaker MT. Adult stem cells in small animal wound healing models. Methods Mol Biol 2013;1037:81-98.

14. Hocking AM. Mesenchymal Stem Cell Therapy for Cutaneous Wounds. Adv Wound Care (New Rochelle) 2012;1: 166-71. 
15. Hanson SE, Bentz ML, Hematti P. Mesenchymal stem cell therapy for nonhealing cutaneous wounds. Plast Reconstr Surg 2010;125:510-6.

16. Chen L, Tredget EE, Wu PY, et al. Paracrine factors of mesenchymal stem cells recruit macrophages and endothelial lineage cells and enhance wound healing. PLoS One 2008; 3:e1886.

17. Behr B, Ko SH, Wong VW, et al. Stem cells. Plast Reconstr Surg 2010;126:1163-71.

18. De Ugarte DA, Alfonso Z, Zuk PA, et al. Differential expression of stem cell mobilization-associated molecules on multi-lineage cells from adipose tissue and bone marrow. Immunol Lett 2003;89:267-70.

19. Ishikawa T, Banas A, Hagiwara K, et al. Stem cells for hepatic regeneration: the role of adipose tissue derived mesenchy- mal stem cells. Curr Stem Cell Res Ther 2010;5:182-9.

20. Gir P, Oni G, Brown SA, et al. Human adipose stem cells: current clinical applications. Plast Reconstr Surg 2012;129: 1277-90.

21. Haddad-Mashadrizeh A, Bahrami AR, Matin MM, et al. Human adipose-derived mesenchymal stem cells can survive and integrate into the adult rat eye following xenotransplantation. Xenotransplantation 2013;20:165-76.

22. Yucel E, Alagoz MS, Eren GG, et al. Use of Adipose-Derived Mesenchymal Stem Cells to Increase Viability of Composite Grafts. J Craniofac Surg 2016;27:1354-60.

23. Oh JS, Ha Y, An SS, et al. Hypoxia-preconditioned adipose tissue-derived mesenchymal stem cell increase the survival and gene expression of engineered neural stem cells in a spinal cord injury model. Neurosci Lett 2010;472:215-9. 